\title{
Liquid array diagnostics: a novel method for rapid detection of microbial communities in single-tube multiplex reactions
}

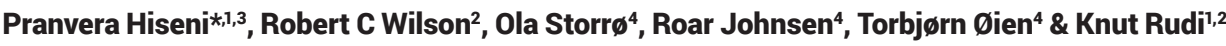

\section{ABSTRACT}

We present a novel liquid array diagnostics (LAD) method, which enables rapid and inexpensive detection of microbial markers in a single-tube multiplex reaction. We evaluated LAD both on pure cultures, and on infant gut microbiota for a 15-plex reaction. LAD showed more than $80 \%$ accuracy of classification and a detection limit lower than $2 \%$ of the Illumina reads per sample. The results on the clinical dataset showed that there was a rapid decrease of staphylococci from 10-day- to 4-month-old children, a peak of bifidobacteria at 4 months, and a peak of Bacteroides in 2-year-old children, which is in accordance with findings described in the literature. Being able to detect up to 50 biomarkers, $L A D$ is a suitable method for assays where high throughput is essential.

\section{METHOD SUMMARY}

Liquid array diagnostics use short DNA duplexes, where one of the oligonucleotides is labeled with a fluorophore and the other, upon the presence of target DNA, becomes labeled with a quencher molecule. The novelty of this method lies in the combination of many duplex melting profiles and several channels of detection on a qPCR instrument, to detect multiple events of fluorescence quenching in a single-tube multiplex reaction.

\section{KEYWORDS:}

fluorescence - liquid array $\cdot$ microbiota - qPCR instrument $\cdot$ quenching

'Department of Chemistry, Biotechnology \& Food Sciences, Norwegian University of Life Sciences, P.O. Box 5003, 1432 Aas, Norway; ${ }^{2}$ Inland Norway University of Applied Sciences, Hamar, Norway; ${ }^{3}$ Genetic Analysis AS, Oslo, Norway; ${ }^{4}$ Department of Public Health and Nursing, Norwegian University of Science \& Technology, Trondheim, Norway; *Author for correspondence: ph@genetic-analysis.com; pranvera.hiseni@nmbu.no

BioTechniques 66: 143-149 (March 2019) 10.2144/ btn-2018-0134
The field of gut microbiota analysis has, until now, been dominated by relatively small-scale explorative studies, with several contradicting findings obscuring the truth in literature [1,2]. We are therefore at a stage where highthroughput, low-cost, targeted approaches are needed in order to generalize knowledge, and to evaluate previous findings. Presently, the GA-map ${ }^{\circledR}$ platform (Genetic Analysis AS) is the only clinically validated tool designated for gut microbiota diagnostics. The GA-map method allows for the faster assessment of the abundance of microbial markers in a sample, compared with NGS techniques [1]. However, it is based on solid-phase hybridization, which creates a bottleneck in sample processing and renders the test relatively expensive.

In this article, we present liquid array diagnostics (LAD), a novel approach for detecting bacterial communities using real-time PCR instrumentation. LAD combines single nucleotide primer extension with high-resolution melting (HRM) in the concept of a liquid array. It does not require physical separation of the probes prior to detection, thus avoiding a bottleneck in sample processing and ensuring rapid results at very low running costs. Requiring only a qPCR instrument, it has great potential for use as a routine tool for diagnostics by reporting multiple gut microbial markers in a single-tube multiplex reaction within a working day. A schematic outline of LAD is provided in Figure 1.

We evaluated LAD both on pure cultures, and on infant gut microbiota. The rationale for investigating the infant gut microbiota is that their composition and development are well described by many studies [2-4], and that we can utilize an already designed and validated GA-map probe set [5]. Furthermore, the development of the gut microbiota during infancy is crucial for health later in life. However, large-scale validation studies are required before knowledge about the gut microbiota can be utilized in clinical practice.

We present results demonstrating the sensitivity and specificity of LAD, in addition to exemplifying its utility on a medium-scale clinical cohort.

Taken together, LAD is a promising method, filling the need for large-scale gut microbiota validation tools.

\section{MATERIALS \& METHODS}

Template generation for labeling probes labeling

We used genomic DNA extracted from 18 different bacterial isolates for PCR amplification. These strains represented targets for one or more labeling probes (LP), thus the purpose was to use them for validation of specificity and reproducibility of our assay. The chosen bacteria were: Gemella sanguinis, Escherichia coli, Salmonella bongori, Salmonella enterica, Salmonella typhimurium, Klebsiella pneumoniae subsp. Pneumonae, Streptococcus pyogenes, Streptococcus pneumoniae, Salmonella enterica subsp. Enterica, Bacteroides vulgatus, Bacteroides fragilis, Bacteroides dorei, Staphylococcus aureus subsp. Aureus, Staphylococcus aureus, Bifidobacterium breve, Bifidobacterium longum, Enterococcus faecalis and Streptococcus sanguinis.

In addition, DNA extracted from 541 PACT (Prevention of Allergy Among Children in Trondheim) study stool samples was utilized in PCRs to generate the templates for LP labeling. These samples were collected from pregnant mothers and their children at up to several post-birth ages. Their 


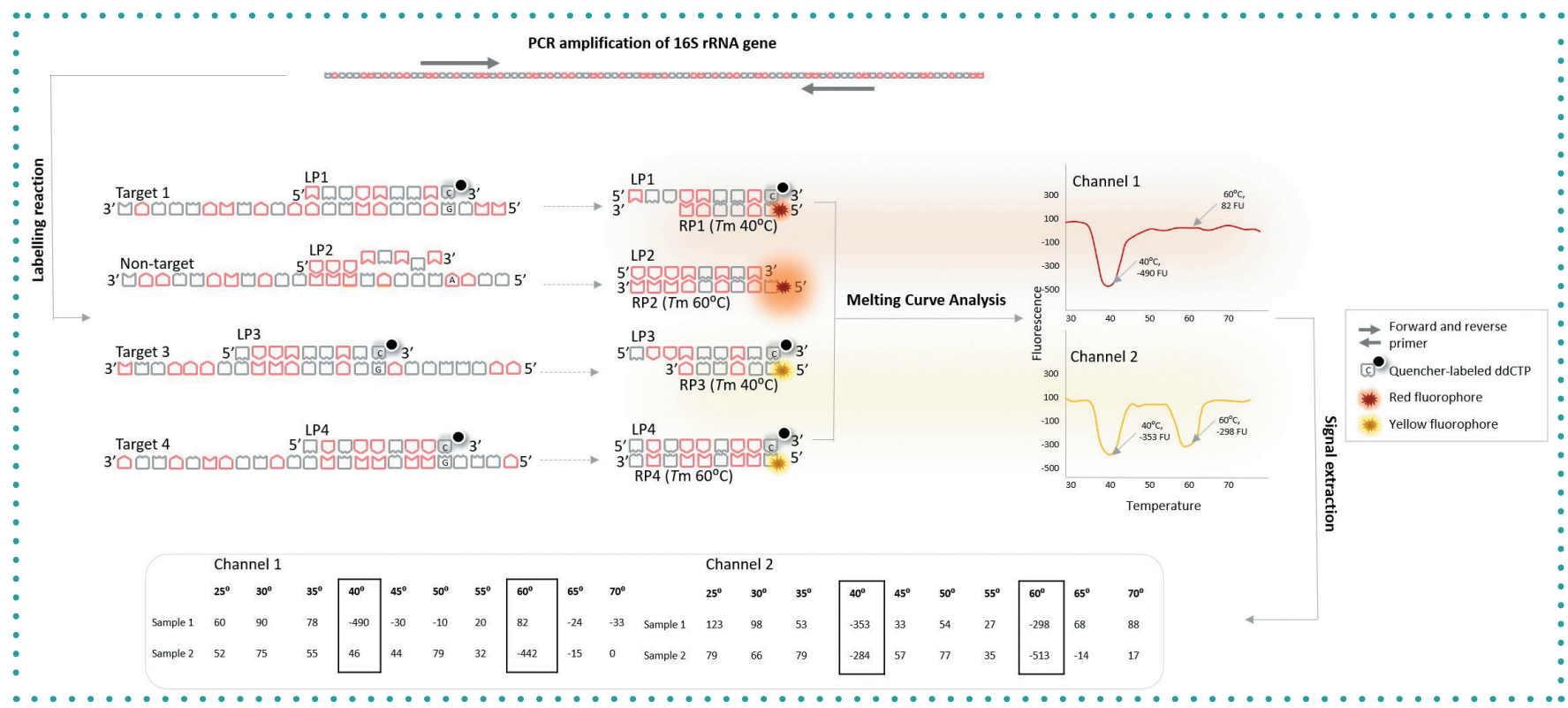

Figure 1. An overview of liquid array diagnostics (LAD) method. The initial step includes PCR amplification of 16S rRNA gene, where each LP is targeted If the target DNA is present, LPs become labeled with a ddCTP conjugated with a quencher molecule. Subsequently, fluorophore-labeled RPs complementary to LPs are added into the solution mix. Upon duplex formation, at a specific melting temperature, the fluorescence of the reporter decreases abruptly. Multiple targets can be detected in a single-tube reaction by combining different duplex melting temperatures and fluorophore colors. In the last step, the derivative fluorescence units (FU) are extracted from each temperature where signals are expected for further data processing. LP: Labeling probe; RP: Reporter probe.

distribution was as following: 110 were collected from pregnant mothers, 122 from children up to 10 days old, 126 samples from 4-month-old children, 89 samples from 1-year-old children, and 94 from 2-year-old children. We used gDNA that was already extracted. The extraction protocol can be found in the Materials and Methods section of Vebø et al. (2011) [5].

A total reaction volume of $25 \mu \mathrm{l}$ comprising $1 \mu$ l bacterial lysate as a source of DNA template, $0.05 \mathrm{U}$ HOT FIREPol ${ }^{\circledR}$ DNA Polymerase, $1 \mathrm{X}$ B1 buffer, $2.5 \mathrm{mM} \mathrm{MgCl}$ (all from Solis Biodyne, Estonia), 0.2 mM dNTPs (Thermo Fisher Scientific, MA, USA), $0.2 \mu \mathrm{M}$ sense primer (Mangala F-1, 5'-TCCTACGGGAGGCAGCAG- $\left.3^{\prime}\right)$, and $0.2 \mu \mathrm{M}$ antisense primer (16SUR, 5'-3' CGGTTACCTTGTTACGACTT) was designed to amplify a segment of 16S rRNA gene. PCR amplification was initiated with a period of $15 \mathrm{~min}$ at $95^{\circ} \mathrm{C}$ to activate the DNA polymerase, followed by 30 cycles, each consisting of 30 s denaturation at $95^{\circ} \mathrm{C}, 30 \mathrm{~s}$ annealing at $55^{\circ} \mathrm{C}$ and an $80 \mathrm{~s}$ elongation at $72^{\circ} \mathrm{C}$ performed using an Applied Biosystems Veriti ${ }^{\prime}$ Thermal Cycler (Life Technologies, CA, USA). A final elongation step of $7 \mathrm{~min}$ at $72^{\circ} \mathrm{C}$ was also included. The amplified products were treated with $2.4 \mathrm{U}$ of Exonuclease I (Exol, Biolabs Inc., MA, USA) and $6.4 \mathrm{U}$ of shrimp alkaline phosphatase (USB Corporation, $\mathrm{OH}$, USA) prior to incubation at $37^{\circ} \mathrm{C}$ for $120 \mathrm{~min}$, and at $80^{\circ} \mathrm{C}$ for $15 \mathrm{~min}$.

\section{Single nucleotide extension of the LPS}

A total reaction volume of $15 \mu \mathrm{l}$ comprising $5 \mu \mathrm{l}$ Exo-SAP-treated template (or water as 'no template' control), LPs at a final concentration of $0.1 \mu \mathrm{M}, 0.8 \mu \mathrm{M}$ ddCTP-ATT0612Q (Jena Biosciences, Germany), $20 \mu \mathrm{M}$ ddTTP, $1 \mathrm{mM} \mathrm{MgCl}{ }_{2}, 1 \mathrm{XC}$ buffer and $0.25 \mathrm{U}$ HOT TERMIPOL ${ }^{\oplus}$ DNA Polymerase (all from Solis Biodyne, Estonia) was prepared. Labeling reactions were performed using an Applied Biosystems Veriti" Thermal Cycler, employing an activation step at $95^{\circ} \mathrm{C}$ for $12 \mathrm{~min}$, followed by 40 cycles, each consisting of $96^{\circ} \mathrm{C}$ denaturation for $20 \mathrm{~s}$ and $60^{\circ} \mathrm{C}$ annealing/elongation for $40 \mathrm{~s}$.

\section{Melting curve analysis}

$5^{\prime}$ fluorescently labeled reporter probe(s) (RP) were added to the LP labeling reactions at a final concentration of $0.005 \mu \mathrm{M}$ each, with the exception of RPs 1_1 RP ROX, 1_2_2 RP ROX, 6_2_2 RP HEX, 6_1_4 RP HEX and 2_4_1 RP FAM, which had a final concentration of $0.02 \mu \mathrm{M}$ each; reagent $\mathrm{S}$, available from INN (Inland Norway University of Applied Sciences, Norway), was also added to a final concentration of $0.1 \%$. The melting curve analysis was performed using a 7500 Fast qPCR instrument (Applied Biosystems, USA) with the following dissociation steps: $95^{\circ} \mathrm{C}$ for $15 \mathrm{~s}, 30^{\circ} \mathrm{C}$ for $1 \mathrm{~min}, 95^{\circ} \mathrm{C}$ for $15 \mathrm{~s}$ and $60^{\circ} \mathrm{C}$ for $15 \mathrm{~s}$. Fluorescence was detected and expressed in dissociation curves as the derivative of the fluorescence versus temperature measurements $(\mathrm{dF} / \mathrm{dT})$ versus temperature (Temp.). Positive signals were observed as negative peaks, representing the abrupt, temperature-dependent drop of fluorescence.

\section{Extraction of peaks \& determination of positive signals for clinical samples}

For the sake of simplicity, all data were multiplied by -1 since originally, positive LAD signals have negative values.

Fluorescence values were extracted from temperature measurements where quenching signals were expected (e.g., the fluorescence value at $67.7^{\circ} \mathrm{C}$ on HEX channel, where UNI probe was designed to quench). In addition, such values were extracted from 5 no template controls (NTC), with the aim of determining the borderline 
Table 1. Probes designed by Genetic Analysis AS for GA-map ${ }^{\circledR}$ array, used as labeling probes by liquid array diagnostics.

\begin{tabular}{|c|c|c|}
\hline Probe identifier & Taxonomic group(s) detected & Probe sequence $\left(5^{\prime}-3^{\prime}\right)$ \\
\hline 1_1 & Bacteroides & TTGCGGCTCAACCGTAAAATTG \\
\hline 1_2_2 & Bacteroides (dorei, fragilis, thetaiotaomicron, vulgatus) & GCACTCAAGACATCCAGTATCAACTG \\
\hline 2_1_min1b & Gamma-proteobacteria & CAGGTGTAGCGGTGAAATGCGTAGAGAT \\
\hline 2_3_2 & Gamma-proteobacteria subgroup & CGGGGATTTCACATCTGA \\
\hline 4_4_2 & Enterococcus, Listeria & TCСААТGАСССТССС \\
\hline 4_5_2 & Streptococcus pyogenes & GATTTTCСАСТСССАССАT \\
\hline 4_6_1 & Streptococcus sanguinis & САСТСТСАСАСССGTT \\
\hline $6 \_1 \_4$ & Bifidobacterium longum & TGCTTATTCAACGGGTAAACT \\
\hline $6 \_2$ & Actinobacteria & CGTAGGCGGTTCGTCGCGT \\
\hline 6_2_2 & Bifidobacterium breve & CGGTGCTTATTCGAAAGGTACACT \\
\hline UNI01 & 16S Universal & CGTATTACCGCGGCTGCTGGCA \\
\hline
\end{tabular}

separating positive signals from background fluorescence. First, we calculated the distance of the observed positive signals from the mean background fluorescence using a standard Z-score. Following that, the margin separating the signals from the background was assigned to be the mean value of NTC plus two-times its standard deviation $(\mu+2 \Sigma)$.

However, a different approach was used to assign positive signals for 5_1_2. Considering that there is a tight melting temperature $(T \mathrm{~m})$ range separating 5_1 from 5_1_2 signals, using the above-mentioned formula would report false-positive signals for Staphylococcus (5_1_2 probe) since the fluorescence measurements at $55.8^{\circ} \mathrm{C}$, where 5_1_2 is designed to quench, are interferingly high for each sample where 5_1 is truly quenched $\left(50.8^{\circ} \mathrm{C}\right)$. Thus, fluorescence values at $55.8^{\circ} \mathrm{C}$ were extracted from eight random samples where only 5_1 was observed to give signal. The mean value of these samples was added with three standard deviations $(\mu+3 \Sigma)$, which was used as a margin to separate the bona fide Staphylococcus signals. All data values higher than the margins were accepted as positives.

\section{Probe design}

The probes, designed by Genetic Analysis AS [5], were used as LPs (Table 1), whereas the RPs were designed to be complementary to the LPs, so that they create duplexes that dissociate at a chosen temperature. Each probe has a code identifier (for example 1_1 for Bacteroides), originally used in Vebø et al. (2011) [5]. The $\mathrm{Tm}$ of the probes was calculated by the Oligoanalyzer 3.1 web-based bioinformatics tool (Integrated DNA Technologies) and target $T \mathrm{~ms}$ were achieved by varying the length of the RPs.

The reporter probes were designed to anneal to the 3 '-end of each respective labeling probe, thus placing the fluorophore, coupled to the terminal 5'nucleotide of the $\mathrm{RP}$, in close physical proximity to the quencher molecule located at the $3^{\prime}$ end of the labeled LP. The list of the reporter probes is presented in Table 2.

\section{Comparison of LAD-based results with} Illumina sequencing data

87 random PACT samples (34 samples of children up to 10-days old, 15 of 4-montholds, 15 of 1-year-olds, 12 of 2-year-olds and 11 of pregnant women) were picked to be sequenced with an Illumina MiSeq System (Illumina, CA, USA). The purpose of this step was to confirm the identities of samples and compare them with the results obtained with the LAD assay, by performing in silico labeling of the reads. In silico labeling was performed by textual mapping of the 'labeled' LPs to the operational taxonomic unit (OTU) DNA sequences retrieved by Illumina, using the Sequence Manipulation Suite: Primer Map tool [6]. All OTUs that were detected by the same probe were grouped together and their number of reads was summed up for each sample. The total number of such reads was then compared with the LAD signal intensity for the said probe. Prior to doing so, LAD data were normalized so that any number below the cut-off value would be equal to zero.

To calculate the specificity and sensitivity, we performed a receiver operator characteristic (ROC) curve analysis (MedCalc Software, Ostend, Belgium), which plots the true positive signals (as determined with $L A D$ ) against the false positives for different cut-off points (the number of Illumina reads). This helped find the optimum copy number of target sequences that can be detected using our method. 
Table 2. Reporter probe sequences.

Reporter probe

\begin{tabular}{|l|l}
\hline 1_1 RP ROX & \\
\hline 1_2_2 RP ROX & \\
\hline 2_1_min1b RP ROX
\end{tabular}

2_3_2 RP ROX

4_1 RP CY5

4_5_2 RP CY5

4_8_1_RP2_CY5

4_6_1 RP CY5

2_4_1 RP FAM

4_4_2 RP FAM

5_1 RP FAM

5_1_2 RP FAM

6_2 RP HEX

6_2_2 RP HEX

6_1_4 RP HEX

UNI01 RP HEX
5'-3' sequence

/56-ROXN/TTTCAATTTTACGG

/56-ROXN/TTTCAGTTGATACTGG

/56-ROXN/TATCTCTACGCATTTCACCGCTACA

/56-ROXN/TTTCAGATGTGAAATCCC

/5CY5/TTTAGTGAAGAAG

/5CY5/TATGGTGGGAGT

/5CY5/TAAGTCTGACCGAGCAACGCCGC

/5CY5/TTAACGGGTGTGAGAGTG

/56-FAM/TAACTGCATTC

/56-FAM/TTTGGGAGGGTCAT

/56-FAM/TTTGTGGCAAGCGTTG

/56-FAM/TTACCTAATCAGAAAGCCACG

/5HEX/TTTTACGCGACG

/5HEX/TTAGTGTACCTTTCG

/5HEX/TTAGTTTACCCGTTGAAT

/5HEX/TTGCCAGCAGCCGCGGTAATACG
- Subsequently, for each probe, the numbers of the Illumina reads lower than LAD detection limit were equated to zero, to test the correlation of the positive signals using Spearman's Rho test.

\section{Statistical analysis}

Minitab Release 15.1.1.0 (Minitab Inc. 2007) was used to perform Student's t-test to compare the differences on quenching strength (fluorescence mean value) between cohorts. For the sake of illustration, the data were normalized so that the cut-off value equals zero. In addition, the differences regarding the prevalence of positive signals were analyzed by using Pearson's chi-squared test.

\section{RESULTS \& DISCUSSION}

Optimization of the LAD-based

microbiota detection assay

Based on pure cultures, we first adjusted the level of probes present in the reaction in order to achieve high signal-to-noise ratios. A detailed description of the experimental setup used in the evaluation is provided in Supplementary Figures S1, S2 and S3. This process was performed empirically (see supplement for details), resulting in an assay that was capable of reporting 15 distin- guishable signals in a one-tube multiplex reaction, consisting of probes reported in Table 1. The signals for each of our probes, besides 6_2 duplex, were at least two standard deviations above the average value of no target reactions, which represented the background noise $(Z>2)$, with a p-value $<0.02$ (Table 3).

The initial evaluation of the assay performance was based on comparisons between experimental and theoretical signals, derived from Vebø et al. (2011) [5]. This analysis showed that the accuracy and specificity of probes was very high, reporting only the target strains in reactions holding individual bacteria or defined bacteria mixtures (Figure 2).

\section{Comparison of LAD-based microbiota assay with Illumina sequencing}

To compare LAD with the output of Illumina sequencing, we sequenced 87 clinical samples, then performed in silico labeling of the retrieved sequences for the nine probes covered by the sequencing amplicon. Subsequently, for each probe we compared LAD signals with the number of sequence copies that acted as a template during in silico labeling. Specifically, we performed ROC curve analysis for each probe to determine accuracy of classification, and to determine limit of detection for the LAD assay. For most of the probes the accuracy of detection, i.e. the number of correct predictions, was high (>80\%). The detection limit for the probes was between 0.2 and $2 \%$, as determined by the percentage of Illumina sequencing reads detected. Furthermore, there was a significant quantitative correlation between Illumina read counts and LAD signals $(p<0.05)$, with Spearman's rho ranging between 0.45 and 0.86 for all the probes (Table 4).

Use of LAD to genotype clinical samples The verified assay was used to probe the microbiota composition from 541 PACT study fecal samples from infants and their mothers.

The highest number of positive signals was reported for 5_1 and 6_2_2 probe duplexes, designed to detect Firmicutes and Bifidobacterium breve, respectively. Overall, the results showed that in terms of prevalence, there is overrepresentation of gammaproteobacteria and Enterococcus/Listeria in 4-month-old children, Bacteroides at 2-years old, Bifidobacterium at 4 months and Staphylococcus in 10-day-old children (Figure 3). 
Table 3. Probe signal-to-noise ratios.

\begin{tabular}{|l|l|l|l|l|l|}
\hline Probe & \multicolumn{2}{l}{$\begin{array}{l}\text { Average of positive } \\
\text { signals }\left(\mu_{1}\right)\end{array}$} & $\begin{array}{l}\text { Average of NT } \\
\text { signals }\left(\mu_{2}\right)\end{array}$ & $\begin{array}{l}\text { NT standard } \\
\text { deviation }(\Sigma)\end{array}$ & \multicolumn{2}{l|}{ Z-score $\left(\left(\mu_{1}-\mu_{2}\right) / \Sigma\right)$ p-value } \\
\hline 6_2 & -287.5 & 70.4 & 87.9 & -4.1 & $>0.99$ \\
\hline UN101 & 1704.1 & -331.1 & 58.2 & 34.9 & $<0.0002$ \\
\hline 6_2_2 & 723.8 & -91.2 & 121.8 & 6.7 & $<0.0002$ \\
\hline 6_1_4 & 2022.9 & -7.2 & 95.9 & 21.2 & $<0.0002$ \\
\hline 4_1 & -537.4 & -2599.6 & 896.6 & 2.3 & 0.01 \\
\hline 4_5_2 & -446.3 & -2398.5 & 826.1 & 2.3 & 0.01 \\
\hline 4_6_1 & -364.9 & -2013.9 & 816.4 & 2.0 & 0.02 \\
\hline 4_8_1 & N/D & & & N/D & N/D \\
\hline 1_1 & 2062.9 & -569.1 & 421.3 & 6.2 & $<0.0002$ \\
\hline 1_2_2 & 2010.5 & -336.4 & 106.5 & 22.0 & $<0.0002$ \\
\hline 2_3_2 & 2230.5 & -326.5 & 234.0 & 10.9 & $<0.0002$ \\
\hline 2_1_min1b & 1865.0 & -473.9 & 83.4 & 28.0 & $<0.0002$ \\
\hline 2_4_1 & 117.9 & -836.5 & 365.8 & 2.6 & 0.0047 \\
\hline 4_4_2 & 1251.5 & -801.7 & 198.5 & 10.3 & $<0.0002$ \\
\hline 5_1 & 1355.9 & -1021.1 & 109.9 & 21.6 & $<0.0002$ \\
\hline 5_1_2 & 2286.7 & -515.5 & 520.8 & 5.4 & $<0.0002$ \\
\hline +The fluorescence values for 4_8_1 probe duplex were not determinedbecause we lacked the DNA template. & \\
\hline N/D: No data; NT: No template. & & & & \\
\hline & & & & \\
\hline
\end{tabular}

The signal strength for $S$. pyogenes and $S$. sanguinis had a peak in 1-year-old children, while the strongest signals for $S$. pneumoniae were in 10-day-old children.

There was no significant change in prevalence for the probe detecting a group of species within Firmicutes (5_1 probe, detecting for Clostridia, Bacillales, Enterococcus and Lactobacillus); however, the signal strength showed an increase parallel with age. The opposite was observed for the other Firmicutes probe, 4_1 (detecting for Lactobacillus, C. perfringens and Staphylococcus), which had a decrease both on prevalence and signal strength in older children.

\section{Use of LAD for rapid detection of microbial communities}

Here we present $L A D$, a novel technique that combines single-nucleotide-extension of the probes with HRM analysis. Compared with existing tools for microbiome testing, LAD-based tests are simpler to perform, are cheaper as they do not require expensive instrumentation and reagents, and yield results faster, within a working day.
Our method does not require a dedicated instrument that would solely be used for LAD-based tests. It requires real-time PCR instrumentation, which is widely and commonly used in most laboratories. In comparison with other real-time PCR-based approaches, it offers a higher level of multiplexity per well, few reagents and short hands-on time, satisfying the actual need of detecting a relatively low number of markers $(<50)$ in a very large number of samples.

LAD represents a highly reproducible method. Initially, the designed probes undergo a process of validation for their specificity, which ensures that all probes become labeled only when their target is present in the reaction. Further on, each labeled probe is tested to ensure it hybridizes only with its corresponding

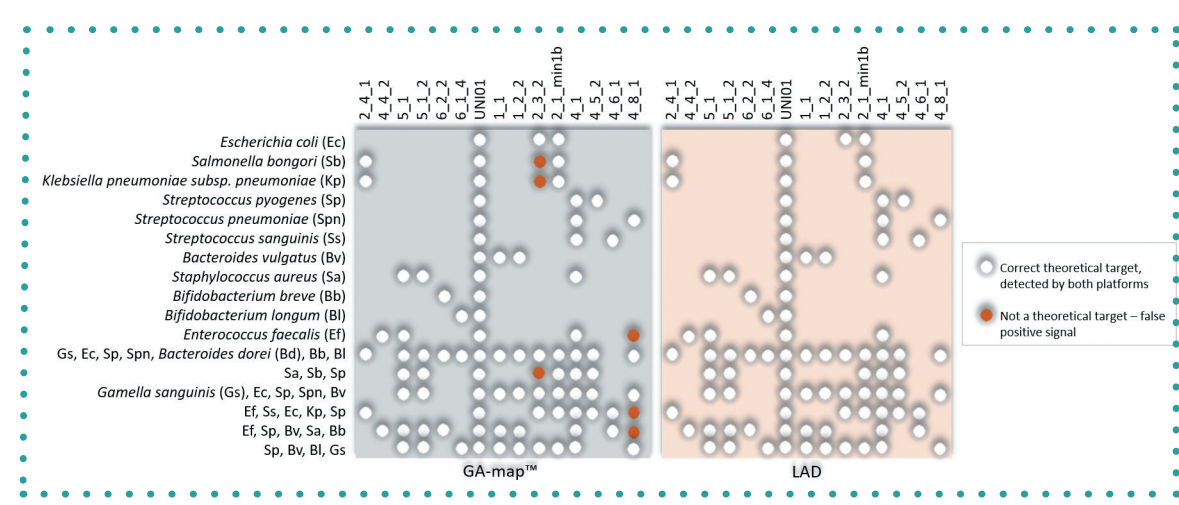

Figure 2. Evaluation of LAD probe accuracy and sensitivity. All signals that were at least two standard deviations away from the background fluorescence were accepted as positives. Tests on individual bacterial strains or defined mixtures of bacteria showed identical results for the correct targets on both platforms, GA-map ${ }^{\circledR}$ (left) and LAD (right). No false-positive signals, reporting nontargets, were registered with $L A D$. 


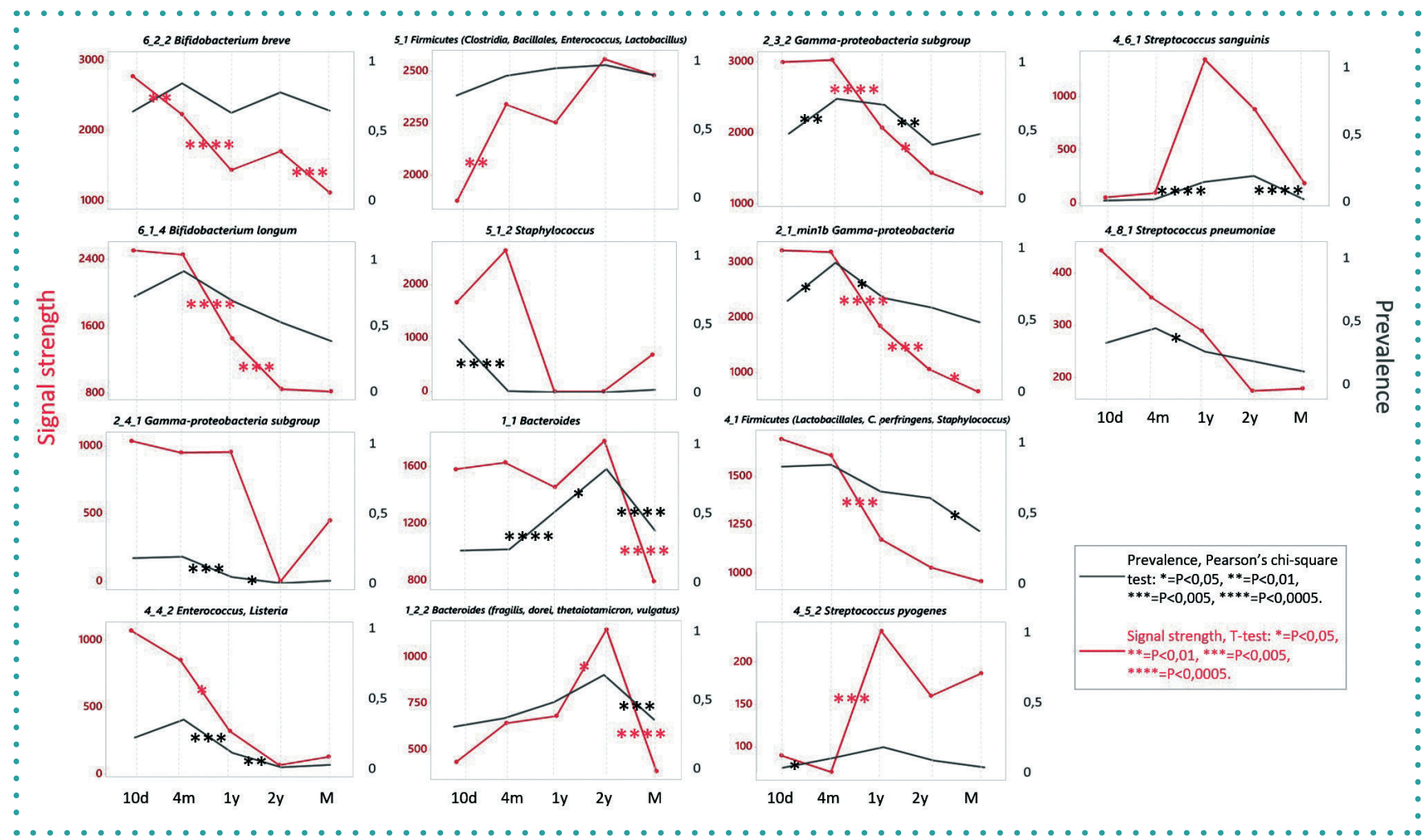

Figure 3. Signal strength and prevalence of positive signals. Significant differences, that here are depicted with *, were observed between groups for most of the probes.

- reporter probe, thus avoiding falsepositive signals being generated in the presence of a nontarget probe.

The addition of a synthetic quencherand-fluorophore-labeled duplex (designated $\mathrm{Tm}$ and detection channel) into the master mix will provide the basis for a well-to-well data normalization, ensuring reproducibility.

For this study, we chose to adopt probes designed by Genetic Analysis AS [5], considering that their platform, GA-map, is an already validated method based on single nucleotide extension. Results obtained with GA-map served as a reference and allowed us to evaluate the overall performance of LAD. We found highly comparable probe specificities using the two technologies, suggesting the transferability of GA-map probes to $L A D$ detection.
Our results on the clinical dataset show that there is a rapid decrease of staphylococci from 10-day- to 4-month-old children, and a peak of bifidobacteria at the age of 4 months, which is in full accordance with the previous findings made with GA-map [5]. However, we identified a peak of Bacteroides in 2-year-old children, whereas Vebø et al. (2011) [5] found that Bacteroides were overrepresented in 4-month-old children. This may be explained by the fact that we did not test an identical set of samples, since an increase of Bacteroides in older children has already been described from many other papers in the literature $[7,8]$.

In addition, we compared our assay with the outcome of Illumina MiSeq sequencing, which demonstrated a high classification accuracy and low detection limit for $L A D$, providing evidence of its sensitivity. The quantitative comparisons, however, showed some more deviations between the two platforms. Unfortunately, we could not Illumina-sequence the $\sim 1200$ bp PCR fragment analyzed with $L A D$ due to the 300-bp limitation in Illumina read-length chemistry, which could potentially explain the differences between the two sets of results.

Numerous gut microbial markers that are linked with many disorders such as obesity [9-11], diabetes [11-13], multiple sclerosis $[14,15]$ or irritable bowel syndrome $[1,16]$ have already been described, but these have not yet been clinically validated in large-scale multicenter studies. With its main advantage of being very cheap, rapid

\section{Table 4. Evaluation of the diagnostic ability of liquid array diagnostics-based tests.}

\begin{tabular}{|l|l|l|l|l|l|l|l|l|l|l|}
\hline Probe & 2_4_1 & 5_1 & 5_1_2 & 1_1 & 1_1_2 & 2_3_2 & 2_1_min1b 4_1 & 4_8_1 \\
\hline Detection limit (\%) & 0.8 & 0.4 & 1.2 & 2.4 & 0.1 & 1.1 & 0.4 & 0.022 & 0.002 \\
\hline Sensitivity (\%) & 90 & 91.9 & 93.3 & 84.8 & 82 & 85 & 65.5 & 62.7 & 69.2 \\
\hline Specificity (\%) & 92.1 & 76.9 & 95.8 & 91.7 & 65.5 & 83 & 86.2 & 85.7 & 68.9 \\
\hline Spearman's Rho & 0.74 & 0.82 & 0.86 & 0.81 & 0.57 & 0.72 & 0.66 & 0.65 & 0.45 \\
\hline
\end{tabular}


and simple, in addition to being an accurate method, LAD will offer this possibility.

We acknowledge the limitations of our method regarding systems where the microbiome composition is complex, unpredictable and constantly shifting. Building a LAD assay de novo is best conducted in systems with relatively low complexity, where the knowledge regarding the microbiome composition is already described, such as is the case with gut microbiota. A well-defined composition is a prerequisite towards designing targeting probes.

Here, we used 15 different probe duplexes, which were designed to utilize four channels of detection and at least three Tms per channel. By using a qPCR machine with six channels of detection and exploiting at least six resolvable Tms per channel, the multiplex level can be elevated to at least a 36-plex. Thus, the possibility of multiplexing is limited by the instrument, and not by LAD technology in itself.

In conclusion, we believe LAD will fulfill the need for assays able to detect up to 50 biomarkers, where high throughput is essential. This will particularly relate to human gut microbiota markers related to health and disease.

\section{AUTHOR CONTRIBUTIONS}

$\mathrm{PH}, \mathrm{RW}$ and $\mathrm{KR}$ conceived and designed the experiments and drafted the work; OS, RJ and $T \varnothing$ helped with the sample collection. All authors contributed equally to analysis and interpretation of data and critical revision of the drafts. All authors approved the final version to be published.

\section{FINANCIAL \& COMPETING INTERESTS DISCLOSURE}

This work was financially supported by Norway Research Council through R\&D project grant no 283783 . The authors have no other relevant affiliations or financial involvement with any organization or entity with a financial interest in or financial conflict with the subject matter or materials discussed in the manuscript apart from those disclosed.

No writing assistance was utilized in the production of this manuscript.

\section{OPEN ACCESS}

This work is licensed under the AttributionNonCommercial-NoDerivatives 4.0 Unported
License. To view a copy of this license, visit http://creativecommons.org/licenses/ by-nc-nd/4.0/

\section{SUPPLEMENTARY DATA}

To view the supplementary data that accompany this paper please visit the journal website at: www.future-science. com/doi/suppl/10.2144/btn-2018-0134

\section{REFERENCES}

1. Casen C,Vebø HC, Sekelja M et al. Deviations in human gut microbiota: a novel diagnostic test for determining dysbiosis in patients with IBS or IBD. Aliment. Pharmacol. Therap. 42(1), 71-83 (2015).

2. Milani C, Duranti S, Bottacini F et al. The first microbial colonizers of the human gut: composition, activities, and health implications of the infant gut microbiota. Microbiol. Mol. Biol. Rev. 81(4), e00036-17 (2017).

3. Dogra S, Sakwinska O, Soh SE et al. Dynamics of infant gut microbiota are influenced by delivery mode and gestational duration and are associated with subsequent adiposity. MBio 6(1), e02419-14 (2015).

4. Rodríguez JM, Murphy K, Stanton C et al. The composition of the gut microbiota throughout life, with an emphasis on early life. Microbial Ecol. Health Dis. 26(1), 26050 (2015).

5. Vebø HC, Sekelja M, Nestestog R et al. Temporal development of the infant gut microbiota in immunoglobulin E-sensitized and nonsensitized children determined by E-sensitized and nonsensitized children determined by
the GA-map infant array. Clin. Vaccine Immunol. 18(8), 1326-1335 (2011)
6. Stothard P. The sequence manipulation suite: JavaScript programs for analyzing and formatting protein and DNA sequences. Biotechniques 28(6), 1102-1104 (2000).

7. Arrieta MC, Stiemsma LT, Amenyogbe N, Brown EM, Finlay $\mathrm{B}$. The intestinal microbiome in early life: health and disease. Front. Immunol. 5, 427 (2014).

8. Cheng J, Ringel-Kulka T, Heikamp-de Jong I et al. Discordant temporal development of bacterial phyla and the emergence of core in the fecal microbiota of young children. ISME J. 10(4), 1002-1014 (2016).

9. Guo X, Li S, Zhang J et al. Genome sequencing of 39 Akkermansia muciniphila isolates reveals its population structure, genomic and functional diverisity, and global distribution in mammalian gut microbiotas. BMC Genom ics 18(1), 800 (2017).

10. Gerard P. Gut microbiota and obesity. Cell Mol. Life Sci. 73(1), 147-162 (2016).

11. Hartstra AV, Bouter KE, Bäckhed F, Nieuwdorp ML. Insights into the role of the microbiome in obesity and Type 2 diabetes. Diabetes Care 38(1), 159-165 (2015).

12. Allin $\mathrm{KH}$, Tremaroli V, Caesar $\mathrm{R}$ et al. Aberrant intestinal microbiota in individuals with prediabetes. Diabetologia 61(4), 810-820 (2018).

13. Delzenne NM, Cani PD, Everard $A$, Neyrinck AM, Bindels LB. Gut microorganisms as promising targets for the management of Type 2 diabetes. Diabetologia. 58(10), 2206-2217 (2015).

14. Chen J, Chia N, Kalari KR et al. Multiple sclerosis patients have a distinct gut microbiota compared to healthy controls. Sci. Rep. 6, 28484 (2016).

15. Miyake S, Kim S, Suda W et al. Dysbiosis in the gut microbiota of patients with multiple sclerosis, with microbiota of patients with multiple sclerosis, with
striking depletion of species belonging to Clostridia XIVa and IV clusters. PLOS One (2015)

16. Bennet SMP, Öhman $L$, Simrén $M$. Gut microbiota as potential orchestrators of irritable bowel syndrome. Gut Liver 9(3), 318-331 (2015).

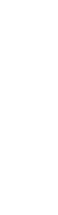

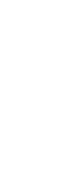

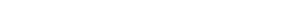

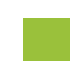

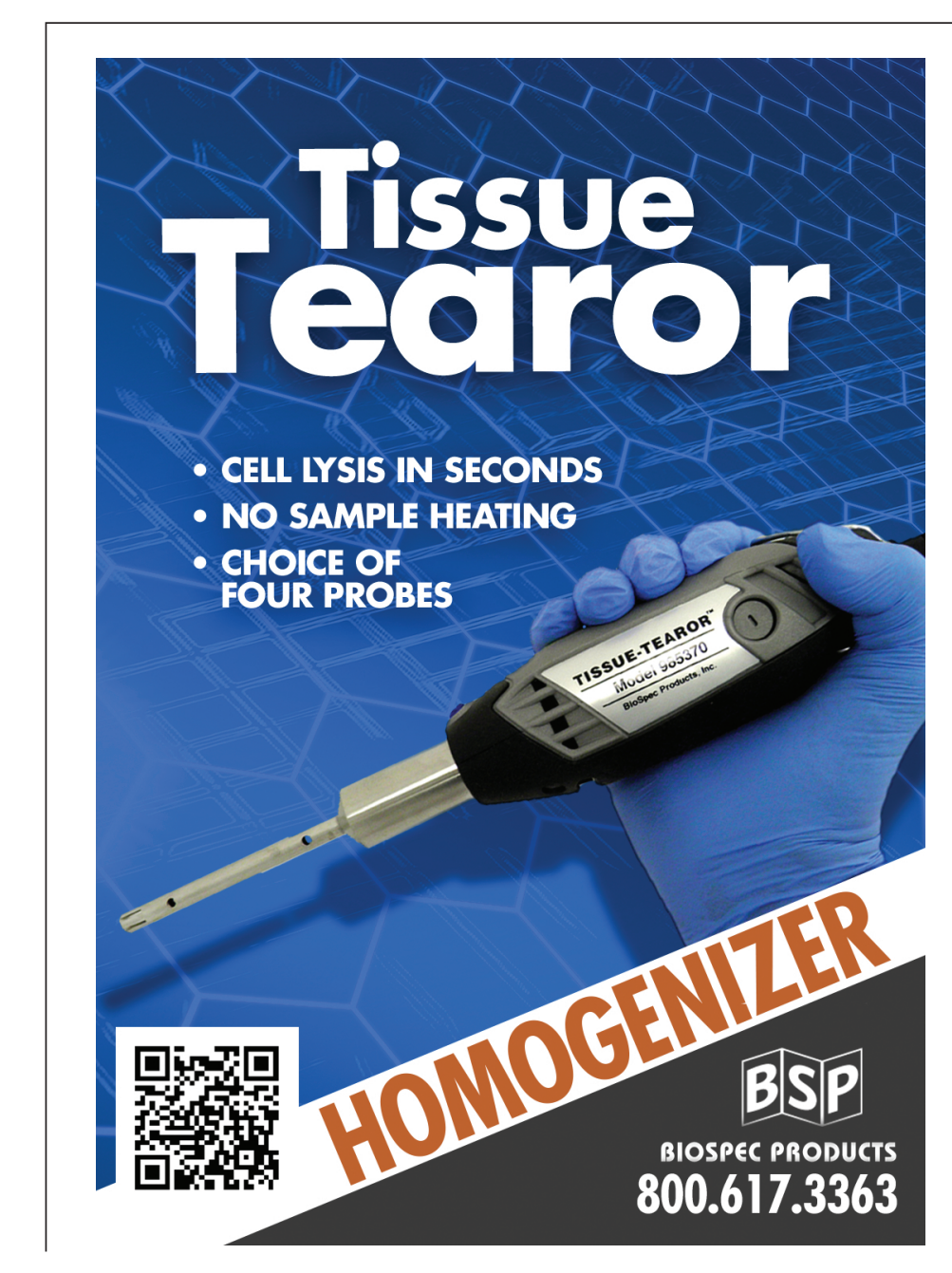

